\title{
Una propuesta para valorar el estado de conservación de los bordes de caminos rurales en el sudeste bonaerense
}

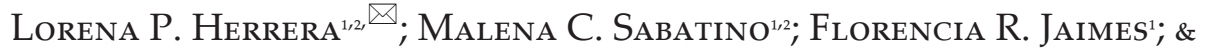 \\ Santiago L. Poggio ${ }^{3 / 4}$ \\ ${ }^{1}$ Unidad Integrada EEA Balcarce, Instituto Nacional de Tecnología Agropecuaria (INTA)-Facultad de Ciencias Agrarias, \\ Universidad Nacional de Mar del Plata, Balcarce, Argentina. ${ }^{2}$ Consejo Nacional de Investigaciones Científicas y Técnicas \\ (CONICET). Argentina. ${ }^{3}$ Universidad de Buenos Aires, Facultad de Agronomía, Cátedra de Producción Vegetal, Buenos Aires, \\ Argentina. ${ }^{4}$ CONICET- Universidad de Buenos Aires, Instituto de Investigaciones Fisiológicas y Ecológicas Vinculadas a la \\ Ecología (IFEVA), Buenos Aires, Argentina.
}

\begin{abstract}
Resumen. Los bordes de caminos rurales (BCR) son elementos clave para conservar la biodiversidad y los servicios ecosistémicos en los paisajes agrícolas. Su restauración y manejo requieren de protocolos sencillos que valoren (diagnostiquen) su estado de conservación y funcionalidad ecológica. En este trabajo elaboramos un índice del valor de conservación (IVC) de los BCR por medio de la construcción de un árbol de decisiones realizado a partir de la consulta a expertos. El índice, que puede asumir valores entre 4 y 30, evalúa el estado de conservación y la funcionalidad ecológica de los BCR según 11 atributos relacionados principalmente con la estructura de la vegetación. El IVC fue aplicado a 30 BCR del sudeste bonaerense (Argentina). Sus valores oscilaron entre 4 y 29. Los BCR cultivados fueron los de menor valor. Los BCR con IVC más alto (>21) se caracterizaron por la mayor riqueza de especies nativas, entomófilas y de valor cultural, la mayor cobertura de especies nativas y pastos perennes, la menor cobertura de malezas y especies invasoras, y la presencia de al menos una especie vulnerable y/o fitotelmata. Este índice representa una primera contribución aplicable al diagnóstico del estado de conservación de los BCR a través de la identificación de atributos claves de la estructura de la vegetación sugeridos por expertos. Además, el índice provee información relativamente sintética sobre el estado de los BCR, los que, pese a sus múltiples funciones ecológicas, rara vez son tenidos en cuenta para su conservación dentro de la planificación territorial.
\end{abstract}

[Palabras clave: biodiversidad, corredores, elementos lineales del paisaje, pastizales remanentes, consulta a expertos, árbol de decisiones]

\begin{abstract}
A proposal to assess the conservation status of roadsides in southeastern Buenos Aires. Rural roadsides (RRS) are key elements for conserving biodiversity and ecosystem services in agricultural landscapes. Their restoration and management require simple protocols to assess (diagnose) their conservation status and ecological functionality. We elaborated a conservation value index (CVI) of RRS by constructing a decision tree based on the experts' opinion. The index, which can range between 4 and 30, evaluates the conservation status and ecological functionality of RRS according to 11 attributes related to vegetation structure. The CVI was applied to 30 RRS in southeastern Buenos Aires (Argentina). Values ranged from 4 to 29, being the cropped RRS those with the lowest value. The RRS with the highest CVI $(>21)$ were those characterized by higher richness of native, entomophily and cultural value species, higher coverage of native species and perennial grasses, lower coverage of weeds and invasive species, and presence of at least a vulnerable and/or phytotelmata species. This index represents a first contribution applicable to the diagnosis of the conservation status of RRS through the identification of key attributes of vegetation structure suggested by experts. In addition, the index provides relatively synthetic information about the status of RRS, which in spite of their multiple ecological functions are seldom taken into account for their conservation within territorial planning.
\end{abstract}

[Keywords: biodiversity, corridors, linear landscape elements, remnant grasslands, consulting expert, decision tree]

\section{INTRODUCCIÓN}

En los paisajes agrícolas, la vegetación que crece en los bordes de los caminos rurales (BCR) representa reservorios importantes de biodiversidad y de provisión de múltiples funciones ecológicas (Hess and Fisher 2001; van der Ree et al. 2015). Dentro de estas funciones se destacan: a) la provisión de hábitat para la

Editora asociada: Marina Omacini flora y la fauna nativa (Burel et al. 1998; Benton et al. 2003; Poggio et al. 2010; Bilenca et al.2012; Torretta and Poggio 2013; Morelli et al. 2014); b) la función como corredores de dispersión para muchas especies vegetales y animales (Saunders and Hobbs 1991; Duelli and Obrist 2003); c) la disponibilidad de hábitat para especies vegetales que son fuente de néctar y 
de polen para numerosos polinizadores (Sáez 2014); d) la provisión de refugio y alimento a los predadores y parásitos de las plagas de los cultivos (Torretta and Poggio 2013); e) el mantenimiento del carbono edáfico (D’Acunto et al. 2014); y f) la filtración de nutrientes y contaminantes desdelotes agrícolas adyacentes (Forman et al. 2003; Tiemeyer et al. 2006). Sin embargo, debido a la estructura lineal de los BCR (elevada relación perímetro-área) y, en consecuencia, su mayor superficie de contacto con la matriz del paisaje, estos ambientes suelen estar sujetos a diferentes presiones ambientales, por lo general derivadas de las actividades humanas. Dentro de estas presiones pueden mencionarse la deriva de las fumigaciones desde los lotes vecinos (Laurance and Yensen 1998), el pastoreo con ganado vacuno, acompañado en algunos casos de quemas de la vegetación para aumentar la receptividad ganadera (observación personal) y el remplazo por cultivos agrícolas en el caso de los bordes suficientemente anchos (observación personal). Por lo expuesto, surge la necesidad de contar con una herramienta que permita valorar el estado de conservación de los BCR, para luego proponer medidas de conservación y manejo que se puedan incorporar a la planificación territorial.

Los indicadores de biodiversidad son atributos biológicos o ambientales cuya medición señala los efectos sobre la biodiversidad de las presiones que causan las actividades humanas (Landsberg and Crowley 2004). La riqueza vegetal, la cobertura del suelo, la diversidad de grupos funcionales de plantas y de especies clave o endémicas, entre otros atributos, son indicadores especialmente importantes de los cambios en la estructura y el funcionamiento de una comunidad, y en consecuencia de su estado de conservación (Burkhard et al. 2012). Por ejemplo, la presencia de especies exóticas e invasoras en una comunidad o ecosistema pueden ser indicadoras de degradación (Groom 2006). En el caso de los BCR, el ancho es otro atributo que puede contribuir tanto positiva como negativamente en la estructura florística y la funcionalidad de las comunidades de estos ambientes (Forman and Godron 1986). Los BCR más anchos no sólo pueden albergar mayor número de especies que los BCR angostos, sino que también es menos probable que el área interior sea afectada por la deriva de las fumigaciones en parcelas vecinas.

Las políticas públicas en materia ambiental son imprescindibles para regular y controlar el uso de los recursos naturales a fin de garantizar la sostenibilidad y el bienestar de la sociedad (Andrade and Vides 2010). En Argentina, el ordenamiento territorial y el observatorio ambiental son dos instrumentos de políticas públicas de implementación reciente (MAGyP 2012). Dentro de estos marcos, en los que las decisiones de conservación o restauración de los ecosistemas naturales presentan cierta urgencia, la consulta a expertos es una estrategia rápida y eficiente para contar con información de calidad sobre un tema específico (Sutherland 2006; Kuhnert et al. 2010; Martin et al. 2012; Barral and Maceira 2012). A su vez, existen diversas herramientas que permiten diagnosticar el estado de conservación de un ecosistema brindando apoyo valioso para la toma de decisiones sobre el monitoreo ecológico. Es el caso del árbol de decisiones, un método que utiliza una serie de reglas basada en nodos (puntos donde una decisión se debe tomar) y ramas (posible alternativa o curso de acción a partir de dicho nodo) (Teli and Kanikar 2015), que permiten organizar en forma jerárquica determinada información (Sharifi et al. 2006). Por ejemplo, a través de la identificación de especies clave en un ecosistema, que por sus funciones sean consideradas favorables (i.e., especies entomófilas) o desfavorables (i.e., especies invasoras), permitiría a los técnicos y científicos conocer su estado de conservación y orientar a los tomadores de decisiones en las medidas para su conservación, restauración y manejo. Así, el desarrollo de un índice para el diagnóstico y valoración de los BCR mediante la generación de reglas de decisiones en base a conocimiento experto puede ser un instrumento muy útil para los planes de ordenamiento territorial.

El sudeste bonaerense es una región con una historia agrícolo-ganadera larga y con un grado elevado de alteración y fragmentación del pastizal original (Bilenca and Miñarro 2004). En algunas zonas, los BCR quedaron como los únicos relictos del pastizal que dominaba antiguamente las Pampas (Vervoorst 1967), aunque la estructura de la vegetación actual es diferente (Vázquez et al. 2014). La preservación y el manejo integral de estos ambientes son cruciales para conservar la biodiversidad y sus funciones dentro del paisaje pampeano. En los últimos años, en esta región se están promoviendo observatorios ambientales e iniciativas de ordenamiento territorial, en los que la valoración de la naturaleza y de los servicios ecosistémicos es un tema que debe ser atendido en forma prioritaria (Maceira et 
al. 2011). Para esto, un aspecto importante a conocer es el estado de conservación o integridad de los BCR en términos de su estructura y funcionamiento (Müller 2005), y a partir de allí promover medidas de restauración y manejo de estos ambientes. Con el objetivo principal de conservar la biodiversidad en los paisajes rurales y de diagnosticar el estado de conservación de los BCR, se realizó una encuesta a expertos sobre los atributos de la estructura y el funcionamiento de la vegetación de los BCR que, a su criterio, permitiría elaborar un índice de valor de conservación (IVC). Sobre la base de las respuestas de los expertos, la información complementaria obtenida de datos empíricos de la vegetación de los BCR del sudeste bonaerense, y el criterio de los autores del presente trabajo, se diseñó un árbol de decisiones (AD) a partir del cual se obtuvo el IVC. Finalmente, se aplicó el IVC a un caso de estudio correspondiente a los BCR del sudeste bonaerense.

\section{Materiales y Métodos}

\section{Diseño de la encuesta y consulta a expertos}

Se elaboró una encuesta que fue enviada a 30 expertos con conocimiento en ecología de plantas y en biodiversidad de la región pampeana. Estos expertos fueron elegidos por su experiencia e idoneidad en la temática, demostrada por sus trayectorias y publicaciones científicas. Los expertos recibieron la encuesta por correo electrónico durante 2015 y 2016. La misma consistió en siete preguntas (Apéndice 1) orientadas a responder: a) ¿qué atributos de la estructura y el funcionamiento de la vegetación de los BCR deberían incluirse como criterios para la elaboración de un índice de valor de conservación?, y b) ¿cuáles serían los valores umbrales de estos atributos para la configuración del índice? La Figura 1 resume el procedimiento metodológico llevado a cabo en este trabajo y en los apartados siguientes se detalla cada paso.

\section{Análisis y síntesis de las respuestas (árbol de decisiones)}

A partir de las respuestas obtenidas en las encuestas recibidas (14 en total) se diseñó un $\mathrm{AD}$ que quedó conformado por una serie de nodos (o puntos de decisión) y ramas (o alternativas de acción), aquíllamados atributos y umbrales, respectivamente. Conforme se van tomando las decisiones a partir de los atributos, el conjunto de alternativas debe ser mutuamente excluyente (i.e., si se selecciona una alternativa, la otra opción queda descartada) y colectivamente exhaustiva (i.e., todas las alternativas posibles deben ser incluidas en el conjunto) (Vélez-Pareja 2003).

En el AD se incluyeron un total de 11 atributos de los BCR, resultado de la opinión de los expertos y la discusión entre los autores del presente trabajo (Tabla 1). Siete de los atributos de la vegetación (riqueza de especies nativas, vulnerables, entomófilas y culturales; abundancia de especies perennes, malezas e invasoras) surgieron de las encuestas y correspondieron a los que recibieron la mayor valoración de los expertos para formar parte del IVC (Tablas 2 y 3). Los valores umbrales

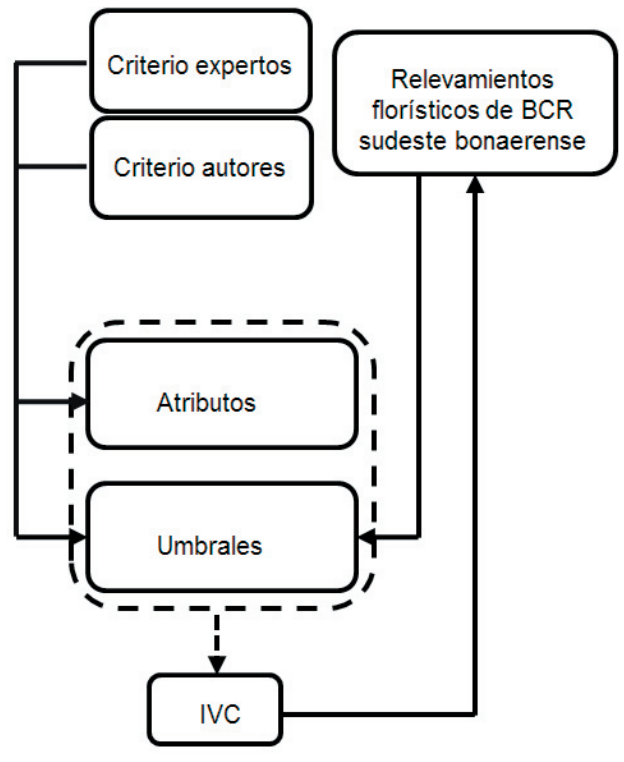

Figura 1. Procedimiento metodológico para la elaboración de un índice de valor de conservación (IVC) de bordes de caminos rurales (BCR). A partir de las respuestas obtenidas en las encuestas realizadas por expertos y de la discusión de los autores de este trabajo se identificaron los atributos y los umbrales que conformarían los nodos y las ramas, respectivamente, de un árbol de decisiones (AD). Además, los datos empíricos obtenidos de los relevamientos florísticos de BCR del sudeste bonaerense (Argentina) contribuyeron a la determinación de algunos valores umbrales. A través de la cuantificación del AD se obtuvo el IVC, que finalmente fue aplicado a los BCR del sudeste bonaerense.

Figure 1. Methodological procedure for the generation of a conservation value index (IVC) of rural roadsides (BCR). From expert's opinion and the discussion of the authors of this work, the attributes and thresholds that would determine the nodes and branches, respectively, of a decision tree (AD) were identified. Also, empirical data obtained from floristic surveys of RRS in the southeast of Buenos Aires (Argentine), allow determining some thresholds value. Through the quantification of the AD the IVC was obtained, which was finally applied to the BCR of southeastern Buenos Aires. 
Tabla 1. Atributos utilizados para la elaboración del árbol de decisiones.

Table 1. Attributes used for the elaboration of the decision tree.

\begin{tabular}{|c|c|}
\hline Atributo & Descripción \\
\hline Ancho & Ancho en metros desde el alambrado hasta el borde de camino o ruta \\
\hline Uso & Uso agrícola o sin uso reconocido \\
\hline Riqueza relativa de especies nativas & $\begin{array}{l}\text { Porcentaje de especies nativas identificadas en el total de especies } \\
\text { listadas en el borde }\end{array}$ \\
\hline Abundancia de especies nativas & Cobertura en porcentaje de especies nativas \\
\hline Presencia de especies vulnerables o en peligro & $\begin{array}{l}\text { Por lo general, especies endémicas con alguna categoría de amenaza } \\
\text { de acuerdo a Delucchi (2006) }\end{array}$ \\
\hline Riqueza de especies entomófilas & Porcentaje de especies de importancia entomófila (Apéndice 2) \\
\hline Riqueza de especies con valor cultural & $\begin{array}{l}\text { Porcentaje de especies con importancia medicinal, forrajera, cultural, } \\
\text { agronómica, ornamental o comestible identificadas en todo el borde }\end{array}$ \\
\hline Presencia de especies fitotelmata o clave & Presencia de al menos una especie fitotelmata o clave (Apéndice 2) \\
\hline Abundancia de especies de malezas & $\begin{array}{l}\text { Cobertura en porcentaje de especies malezas según Richardson et al. } \\
\text { (2000) (Apéndice 2) }\end{array}$ \\
\hline Abundancia de especies invasoras & $\begin{array}{l}\text { Cobertura en porcentaje de especies invasoras según Richardson et al. } \\
\text { (2000) (Apéndice 2) }\end{array}$ \\
\hline Abundancia de especies perennes & Cobertura en porcentaje de especies perennes \\
\hline
\end{tabular}

Tabla 2. Valoración en un rango de 1 a 5 de los atributos de la vegetación por parte de los expertos encuestados. Se muestra la moda y el porcentaje de respuestas correspondiente a cada atributo evaluado. * Sólo para la función ecológica de festuca la valoración fue de -2 (muy negativa) a 2 (muy positiva).

Table 2. Vegetation attributes valuation (range from 1 to 5 ) by the interviewed experts. The mode and the percentage of answers corresponding to each assessed attribute are shown. * Only for the ecologic function of festuca, the valuation was from -2 (very negative) to 2 (very positive).

\begin{tabular}{lll}
\hline Atributos evaluados & Moda & Respuestas (\%) \\
\hline Riqueza de la vegetación & 3 & 86 \\
Total de especies & 5 & 93 \\
Especies nativas & 3 & 79 \\
Especies entomófilas & 4 & 86 \\
Especies de importancia cultural & 5 & 86 \\
Especies con alguna categoría de amenaza (vulnerable, en peligro, etc.) & \\
Formas de vida & & 64 \\
Dicotiledóneas herbáceas & 5 & 79 \\
Gramíneas cespitosas & 3 & 64 \\
Gramíneas formadoras de mata & 5 & 64 \\
Leñosas & 5 & 57 \\
Función ecológica de Festuca arundinacea* & & 57 \\
Secuestro de carbono & & 57 \\
Reducción del riesgo de erosión del suelo & 1 & 54 \\
Infiltración y retención del agua de lluvia & 2 & 57 \\
Potencial como especie invasora & 1 & -2 \\
Refugio de fauna autóctona & 1 & 57 \\
Otros (especifique) & -2 & \\
\hline
\end{tabular}


Tabla 3. Porcentaje de respuestas del total de expertos que votaron distintos niveles de importancia sobre incluir la cobertura de especies perennes, malezas e invasoras como atributos en el árbol de decisiones.

Table 3. Percentage of responses from total experts who voted for different levels of importance about including perennial, weed and invasive species as attributes in the decision tree.

\begin{tabular}{lccc}
\hline Importancia & Perennes & Malezas & Invasoras \\
\hline Máxima & 25 & - & - \\
Alta & 66.7 & 35.7 & 35.7 \\
Media & 8.3 & 35.7 & 42.8 \\
Baja & - & - & - \\
Ninguna & - & - & - \\
Nro. de expertos & 12 & 14 & 14 \\
\hline
\end{tabular}

en términos de porcentaje para cuatro de estos atributos (riqueza de especies nativas, vulnerables, entomófilas y culturales) fueron definidos a partir de la opinión de los expertos (Tabla 4). Es decir, el porcentaje de riqueza más votado para cada grupo de especie fue considerado como valor umbral. Por ejemplo, 5 de los 12 expertos que dieron su opinión al respecto, consideraron que el valor umbral para la riqueza de especies nativas debería ser del 50-75\% (Tabla 4). Por otro lado, los valores umbrales para los demás atributos de la vegetación (abundancia de especies perennes, malezas e invasoras) fueron definidos a partir de la información empírica obtenida de los bordes de camino del sudeste bonaerense (ver "Aplicación del índice de valor de conservación a un caso de estudio"), ya que los mismos no fueron consultados en esta versión de la encuesta. De los cuatro atributos restantes, respecto a los 11 totales, tres surgieron por el criterio de los autores (ancho del BCR, uso cultivado vs. no cultivado y presencia de especies clave o fitotelmatas), y uno (abundancia de especies nativas) fue incorporado por sugerencia de los encuestados (Tabla 1). El ancho de BCR se utiliza como indicador de la disponibilidad de hábitat para distintas especies. Sin embargo, los BCR más anchos suelen ser cultivados o incorporados al área de cultivo cuando se eliminan los alambrados. En la zona correspondiente al caso de estudio de este trabajo se observaron BCR cultivados a partir de un ancho mínimo de $5 \mathrm{~m}$, razón por la cual se seleccionó esta medida como valor umbral. Además, incluimos en el AD un nuevo atributo ("uso agrícola") que contempla si el BCR es cultivado o no. Las especies clave o fitotelmatas fueron incorporadas al AD por su importancia ecológica y funcional en el sistema (Apéndice 2). La conservación de estas especies y sus hábitats está estrecha- mente asociada con la preservación de muchas otras especies vegetales y animales (Kitching 2000). En ambientes del sudeste bonaerense, el género Eryngium es un ejemplo típico de especie fitotelmata (Campos 2010), ya que el agua acumulada en sus hojas basales conforma un ambiente propicio para el desarrollo de insectos y otros animales (Elizalde and Lallana 2000). Además, las especies de Eryngium cumplen un rol clave en el mantenimiento de las redes de polinización en pastizales de la región pampeana (Sabatino et al. 2015).

\section{Conformación del índice de valor de conservación}

Una vez establecidos los atributos y los umbrales del AD se procedió a cuantificarlo para obtener el IVC. A cada uno de los atributos (nodos) se le asignó un número (aquí llamado peso) del -1 al 4, en función del valor umbral correspondiente para cada caso de estudio. Estos pesos surgen del análisis de las respuestas de los encuestados en relación con los atributos y los valores umbrales sugeridos (Tabla 2), así como también del criterio de los autores de este trabajo. De esta manera, los atributos que recibieron mayor valoración por los expertos para conformar el IVC recibieron más peso (máximo 4) en relación a los atributos menos valorados en la encuesta, que reciben un peso menor (máximo 2 ó 1). Luego, en función de los valores umbrales para cada atributo en el borde a evaluar, se le asigna el peso máximo o mínimo correspondiente para cada caso en el AD. Por ejemplo, para el atributo "riqueza de especies nativas" se le asignó un valor umbral del $50 \%$, por lo que si en el borde estudiado la riqueza de especies nativas es mayor a ese valor, le corresponde un peso de 4 , mientras que se le asigna un peso de 1 si es menor. De esta manera, a partir de la sumatoria de los pesos correspondientes para cada atributo en el AD se obtiene el IVC para cada uno de los BCR.

\section{Aplicación del índice de valor de conservación a un caso de estudio}

El IVC fue aplicado a $30 \mathrm{BCR}$ distribuidos de a pares a cada lado del camino y ubicados dentro de una de las áreas de monitoreo del sitio piloto Sudeste Bonaerense del Observatorio Nacional de Degradación de Tierras y Desertificación, denominada

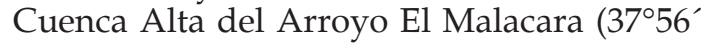


$\left.\mathrm{S}-58^{\circ} 21^{\prime} \mathrm{O}\right)$. La cuenca, con una superficie de $185 \mathrm{~km}^{2}$, se ubica en el partido de Balcarce (provincia de Buenos Aires), dentro de la subregión Pampa Austral (Figura 2). Los suelos son principalmente Argiudoles Típicos y Paleustoles Petrocálcicos (INTA 1991). El clima es templado húmedo, con precipitación media anual de $800 \mathrm{~mm}$ y temperatura media anual de $14^{\circ} \mathrm{C}$. La vegetación original consistía en pastizales dominados por especies de los géneros Nasella, Piptochaetium, Bromus, Aristida, Briza y Setaria; pajonales de Paspalum quadrifarium y arbustales de Eupatorium spp., Colletia spp. y Bacharis spp., entre otras especies (Frangi 1975; Soriano et al. 1991). Con el tiempo, esta vegetación fue remplazada por cultivos anuales y pasturas perennes (Viglizzo et al. 2001). En la actualidad, la encontramos representada en los ambientes no arables como las sierras, los bordes de caminos y los terraplenes de vías férreas, aunque con distinto estado de conservación (Herrera and Laterra 2011; Sáez et al. 2014). Una característica de los bordes de caminos y rutas actuales del sudeste bonaerense es la presencia de Festuca arundinacea (festuca), una gramínea perenne de crecimiento otoño-inverno-primaveral, introducida en Argentina a mediados del siglo
Tabla 4. Valores umbrales de riqueza (VUR), expresados como rangos de porcentaje, para los distintos atributos de la vegetación que recibieron más votos por los expertos (NE: número de expertos que votaron).

Table 4. Richness threshold values (VUR), expressed as percentage range, for the different vegetation attributes that were more voted by experts (NE: number of experts who voted).

\begin{tabular}{lcc}
\hline Atributos de la vegetación & VUR (\%) & NE \\
\hline Especies nativas & $50-75$ & 5 de 12 \\
Especies entomófilas & $50-75$ & 5 de 12 \\
Especies de importancia cultural & $25-50$ & 4 de 10 \\
$\begin{array}{l}\text { Especies con alguna categoría de } \\
\text { amenaza (e.g., vulnerable, en peligro, etc.) }\end{array}$ & $1-25$ & 8 de 14 \\
\hline
\end{tabular}

XX para incrementar la producción forrajera (Scheneiter et al. 2015). Sin embargo, en los últimos años, la festuca colonizó y dominó los bordes de caminos, favorecida por la simbiosis con un hongo endófito (Epichlöe coenofialum) que a las plantas le confiere mayor vigor, competitividad y tolerancia al estrés hídrico (Belesky and Fedders 1996). Estas características contribuirían a que festuca sea la especie dominante en las comunidades, con el riesgo de que excluya a otras especies vegetales presentes en los bordes (Arachevaleta et al. 1989).
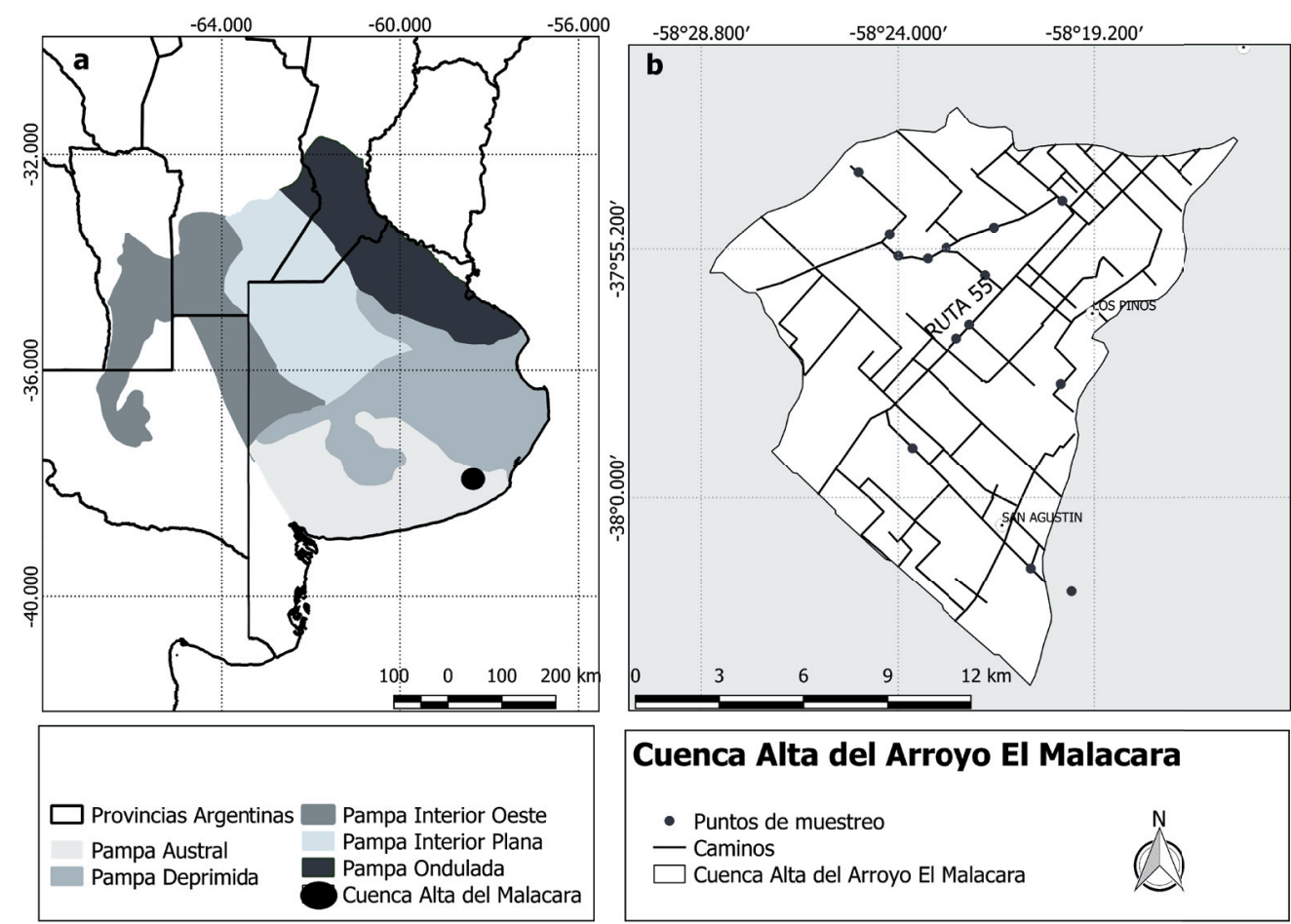

\section{Cuenca Alta del Arroyo El Malacara}

\section{- Puntos de muestreo \\ - Caminos \\ $\checkmark$ Cuenca Alta del Arroyo El Malacara}

Figura 2. Ubicación geográfica de la Cuenca Alta del Arroyo El Malacara, en la provincia de Buenos Aires (a), y distribución de los puntos de muestreo dentro de la cuenca (b). Cada punto representa dos sitios de muestreo, que fueron ubicados a ambos lados del camino. Un punto de muestreo fue tomado fuera de los límites de la cuenca.

Figure 2. Geographic location of El Malacara Stream Basin in Buenos Aires province (a), and distribution of sampling sites within the basin (b). Each point represents two sampling sites, which were located at both side of the road. One sampling point appears beyond the limits of the basin. 
Relevamiento florístico y caracterización de bordes de caminos rurales

Los relevamientos florísticos de los 30 bordes de caminos fueron realizados en la primavera de 2013 por medio de transectas ubicadas en las partes planas y con pendiente de cada BCR $\left(50 \times 2 \mathrm{~m}=100 \mathrm{~m}^{2}\right)$ y en el límite con el alambrado $\left(50 \times 1 \mathrm{~m}=50 \mathrm{~m}^{2}\right)$. Dado que el par de bordes a cada lado de un camino puede presentar diferente estado de conservación y uso, se consideran como diferentes e independientes desde el punto de vista biológico. Se registró la identidad y la abundancia (como porcentaje de cobertura de la transecta) de cada especie (Mueller-Dombois and Ellenberg 1974). Las especies vegetales se identificaron mediante el uso de claves correspondientes a la Flora de la Provincia de Buenos Aires (Cabrera 1963, 1965 a y b, 1967, 1968 y 1970; Flora Argentina 2012 [www.floraargentina.edu.ar]) y fueron agrupadas de acuerdo a su origen (e.g., nativas y exóticas), a la forma de vida (e.g., hierbas anuales, perennes y leñosas), endemismos, potencial entomófilo, claves o fitotelmatas, malezas, invasoras y por su valor como forrajera, medicinal, comestible y ornamental de acuerdo a distintos reportes bibliográficos (Montes et al. 2001; Alonso and Peretti 2006; Rapoport et al. 2009; Roitman and Preliasco 2012). Además, se midió el ancho de los BCR desde el alambrado hasta el borde del camino o ruta.

\section{RESULTADOS Y DISCUSIÓN}

\section{Conformación del árbol de decisiones y del indice de valor de conservación}

Los dos atributos que caracterizarían un mejor estado de conservación de la vegetación y que fueron mejor valorados por los encuestados, según la moda y el porcentaje de respuestas, son "riqueza de las especies nativas" y "presencia de especies con alguna categoría de amenaza" (moda 5 para ambos, 93\% y 86\% porcentaje de respuestas, respectivamente) (Tabla 2). Los valores umbrales más votados para la riqueza de ambos grupos fueron 50$75 \%$ y $1-25 \%$, respectivamente (Tabla 4 ), y se debe destacar que una proporción baja de los encuestados incluyó en sus respuestas la sugerencia de valores umbrales. Dado que el valor umbral más votado para las especies con alguna categoría de amenaza fue el más bajo del listado de rangos proporcionado en la encuesta (Tabla 4), para su simplificación se transformó en una variable discreta de presencia o ausencia (1- 0 , respectivamente).
Es decir, con que al menos una especie de este tipo se encuentre en el borde estudiado es suficiente para darle el peso correspondiente en el AD. Este mismo criterio se utilizó para definir el valor umbral del atributo "presencia de especies clave o fitotelmatas". Algunos expertos sugirieron la inclusión de atributos relacionados con la abundancia, asumiendo que las especies más abundantes controlan el funcionamiento del sistema. Por ejemplo, desde una perspectiva ornitológica se destacó que ciertas especies vegetales típicas del pastizal, por su dominancia y estructura confieren refugio y sitios de nidificación para las aves. Así, por criterio de los autores del presente trabajo se decidió incorporar al índice el atributo "cobertura de especies nativas" por ser las especies nativas uno de los grupos de especies mejor valorados por los encuestados (Tabla 2). Para este atributo se consideró un valor umbral de $40 \%$, de acuerdo a la cobertura promedio de especies nativas halladas en los BCR del sudeste bonaerense, correspondiente al caso de estudio del presente trabajo.

En respuesta a la pregunta de incluir el atributo "cobertura de especies perennes" en la conformación del IVC, ocho de 12 expertos (dos expertos no respondieron a la pregunta 3) lo consideraron muy importante (Tabla 3), principalmente por su importancia en la conservación del suelo y la retención e infiltración del agua de lluvia. Entre los comentarios de los encuestados respecto a esta pregunta, se destacó el rol de las especies perennes en favorecer la estabilidad del sistema en épocas de sequía, ya que impiden la implantación de dicotiledóneas herbáceas exóticas. El valor umbral correspondiente al atributo "abundancia de especies perennes" en el AD fue de 75\%. Este valor surge de la cobertura promedio de especies perennes hallada en BCR del sudeste bonaerense, ya que en esta versión de la encuesta no se consultó a los expertos sobre los valores umbrales de este grupo, sino sólo respecto a su inclusión o no. Otro comentario por parte de un encuestado respecto a incluir la cobertura de las especies perennes en el IVC, menciona la importancia de contemplar en el atributo "la mayor cantidad de especies que integraban el pastizal original". Este es un punto importante a tener en cuenta, ya que en esta parte de la encuesta se consulta por la importancia de las especies perennes en general, tanto nativas como exóticas. Sin embargo, muchas de las especies perennes que se vuelven dominantes en los BCR y que 
pueden cumplir funciones relevantes en el mantenimiento de estos sistemas, no forman parte de la vegetación original (en este caso, el pastizal pampeano). Este es el caso de festuca, que cuando fue consultado sobre su rol en los $\mathrm{BCR}$, los expertos valoraron tanto positiva como negativamente su presencia en estos ambientes, ya sea por su función en reducir el riesgo de erosión como por su capacidad de invasión, respectivamente (Tabla 2). Es decir, un atributo de esta especie es su capacidad de desarrollar abundante biomasa aérea (Scheneiter 2005), lo que le permite mantener la cobertura del suelo, pero a su vez impide que otras especies se establezcan y desarrollen. Para discernir entre estos dos aspectos, esta especie puede formar parte de dos atributos: "cobertura de especies perennes", en la que si es mayor a 75\% suma en el IVC, y "cobertura de especies invasoras", en la que si es mayor a $25 \%$ resta en el IVC.
El atributo "cobertura de las especies malezas" fue considerado de importancia media a alta por 10 expertos, mientras que la "cobertura de las especies invasoras" fue considerada de importancia alta y máxima por 11 expertos (Tabla 3), en ambos casos como indicadores de la funcionalidad de los BCR. Como valor umbral de la "cobertura de las especies de malezas" se utilizó la cobertura promedio de este grupo (40\%) hallada en los BCR del sudeste bonaerense. Mientras que para el atributo "cobertura de especies invasoras" se utilizó como referencia el valor porcentual de constancia (frecuencia de aparición en bordes del sudeste bonaerense) de F. arundinaceae (75\%), por ser una especie invasora clave en estos sistemas.

La conformación del AD resultante en base a los criterios más votados por los expertos, junto con otros criterios propuestos "a posteriori" y
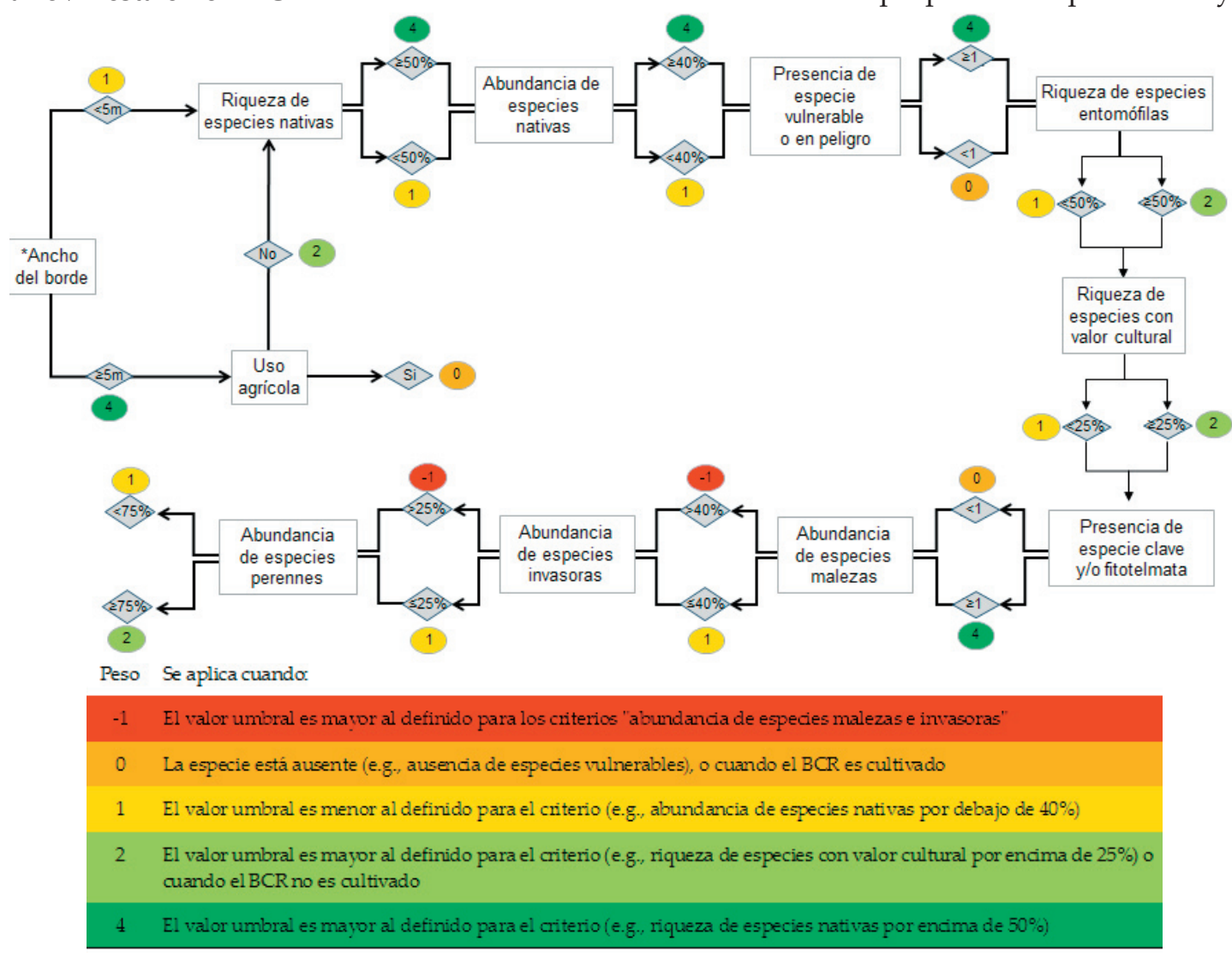

Figura 3. Árbol de decisiones para la elaboración de un índice de valor de conservación de bordes de caminos rurales (BCR). Las cajas representan los nodos o atributos del árbol, las flechas indican las ramas o umbrales y los números dentro de los círculos representan los pesos (-1 a 4) establecidos para cada atributo dependiendo de si el umbral considerado se manifiesta o no en el borde evaluado. Para más detalles, ver "Análisis y síntesis de las respuestas" y "Conformación del índice de valor de conservación". *indica el punto de arranque.

Figure 3. Decision tree for the elaboration of a conservation value index of rural roadsides (BCR). The boxes represent the attribute or nodes of the tree, the arrows indicate the branches or thresholds and the numbers within the circles represent the weight (-1 to 4) established for each attribute depending on whether or not the threshold considered is expressed in the evaluated roadside. For more details, see "Analysis and synthesis of the answers" and "Conformation of conservation value index". *Indicates the start point. 
sobre la base del estudio empírico realizado por los autores de este trabajo, se representa en la Figura 3. Consideramos darle un peso alto (4) a los atributos "presencia de especies nativas" (tanto por su riqueza como por su cobertura), "presencia de especies vulnerables o en peligro de extinción", y "presencia de especies clave o fitotelmatas", de acuerdo a la valoración realizada por parte de los encuestados para ser incluidos dentro del AD (Tabla 2). También le otorgamos un peso alto al atributo "ancho del BCR" porque lo consideramos un indicador de la disponibilidad de hábitat para especies animales y vegetales; así, cobra gran relevancia en un paisaje dominado por la agricultura. Dependiendo de las características del BCR estudiado, el IVC puede asumir valores entre 4 y 30 y, a nuestro criterio, podría desglosarse en tres categorías de valores en función de su división en tres percentiles iguales: IVC bajo (menor al percentil 33), correspondiente a los valores entre 4 y 10 ; IVC medio (mayor al percentil 33 y menor al percentil 66), correspondiente a los valores entre 11 y 20, e IVC alto (mayor al percentil $66)$, correspondiente a los valores entre 21 y 30 (Figura 4).

\section{Aplicación del índice de valor de conservación a un caso de estudio}

Los 30 BCR estudiados presentaron un ancho variable entre 2.5 y $30 \mathrm{~m}$. La caracterización florística indicó la presencia de 110 especies pertenecientes a 91 géneros y 28 familias botánicas. La familia botánica más representada fue Poaceas, que incluyó 35 especies de 25 géneros, seguida por la familia Asteracea, con 22 especies de 19 géneros. Del total de los ejemplares identificados hasta el nivel de especie, 50\% fueron nativas y 59\% fueron perennes. En cuanto a la forma de crecimiento, las dicotiledóneas herbáceas presentaron el mayor porcentaje (69\%),

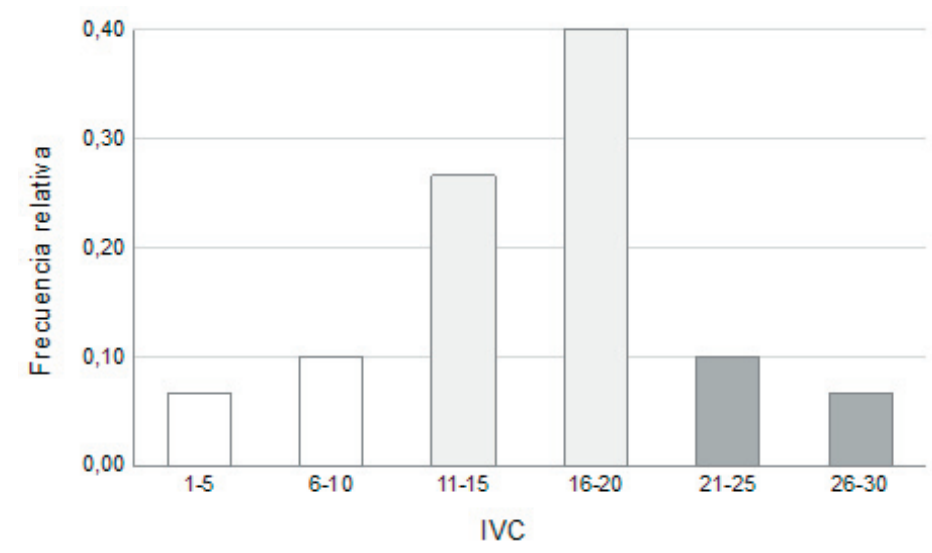

seguidas por las gramíneas (31\%). Doce de las especies nativas registradas fueron endémicas. La gramínea F. arundinacea fue encontrada en $80 \%$ de los bordes estudiados, con coberturas entre 10 y 100\%. El 45\% de las especies identificadas correspondió a individuos con importancia forrajera (24\%), medicinal (17\%), comestible ( $2 \%$ ) y ornamental (2\%). El 42\% de las especies fueron malezas (i.e., Conyza bonaerensis, Centarurea diffusa), mientras que dos especies son consideradas invasoras (F. arundinaceae y Cynodon dactylon), según Richardson et al. (2000). Por otro lado, se registró una especie y un género con características de fitotelmata (Dipsacus fullonum, Eryngium sp.). Cerca de $70 \%$ de las herbáceas de hoja ancha correspondieron a especies con potencial entomófilo.

El rango de valores del IVC aplicado a los BCR del caso de estudio osciló entre 4 y 29 (Figura 4), y los BCR cultivados fueron los de menor valor $(n=2)$. Más de la mitad de los BCR estudiados presentaron valores medios del IVC (entre 11 y 20). Estos bordes se caracterizan por la presencia de especies de importancia entomófila y de valor cultural por encima de los valores umbrales esperados y por la presencia de al menos una especie fitotelmata. Sin embargo, el valor de IVC fue afectado tanto por la baja riqueza y cobertura de especies nativas como por la elevada cobertura de malezas y especies invasoras. Estas últimas características podrían ser consecuencia de los cambios en el uso de la tierra registrados en los últimos años en la región (Paruelo et al. 2006; Bilenca et al. 2009), responsables de la modificación general del paisaje rural, del incremento de agroquímicos en el medio (Oesterheld 2008) y de la degradación de la vegetación nativa. Esto se hace más evidente en los BCR debido a su estructura lineal y angosta, que los hace más susceptibles de recibir la deriva de las fumigaciones de los

Figura 4. Distribución de frecuencias del índice de valor de conservación (IVC) calculados para bordes de caminos rurales de la Cuenca Alta del Arroyo El Malacara, provincia de Buenos Aires. Se discrimina el IVC en valores bajos, medios y altos, según las barras de color blanco, gris claro y gris oscuro, respectivamente.

Figure 4. Frecuency distribution for the conservation value index (IVC) calculted for rural roadsides in El Malacara Stream Basin, Buenos Aires province. The IVC is discriminated in low, medium and high values, according to the white, light gray and dark gray bars, respectively. 
lotes vecinos, con el consecuente impacto sobre la estructura de la vegetación asociada (Poggio et al. 2010). Bajo estas condiciones, los cambios más significativos son la reducción notable de la biomasa y la cobertura del suelo, la disminución de especies nativas y el incremento de especies exóticas, muchas de ellas reconocidas como malezas de cultivos anuales (Chaneton et al. 2002). Así, los BCR muchas veces son considerados reservorios de malezas (Gelbard and Belnap 2003). Sin embargo, es importante destacar que una especiemaleza deun cultivononecesariamente desempeñaría esa función en los bordes. En estos ambientes, muchas especies de malezas contribuyen al mantenimiento de poblaciones de insectos polinizadores y controladores biológicos de plagas de cultivos, a través del ofrecimiento de recursos alimenticios y de refugio (Torreta and Poggio 2013; Sáez et al. 2014).

Sólo cinco BCR presentaron valores altos del IVC (>21) (Figura 4). Estos bordes se caracterizan por la mayor riqueza de especies nativas, entomófilas y con valor cultural, la alta cobertura de especies nativas y de pastos perennes, la baja cobertura de malezas y especies invasoras, y por la presencia de al menos una especie vulnerable, fitotelmata o clave. Algunas de estas características asemejan a estos ambientes al pastizal original que dominaba antiguamente la región, que se caracterizaba por una dominancia elevada de especies de gramíneas nativas perennes, y baja o nula proporción de especies exóticas o invasoras (Vervoorst 1967). Así, estos BCR representan casos de estudio interesantes para profundizar en los factores que definen la estructura, dinámica y conservación de las comunidades de los BCR en el paisaje rural.

\section{CONCLUSIONES}

Este trabajo presenta una herramienta sencilla y novedosa que, por medio de unos simples pasos permite: a) diagnosticar el estado de conservación de los BCR a través de la identificación de atributos claves de la estructura de la vegetación avalados por expertos, y b) contribuir con información sobre elementos del paisaje que, a pesar de sus múltiples funciones ecológicas, rara vez son tenidos en cuenta en la toma de decisiones sobre la conservación de los recursos naturales de un territorio. A su vez, la aplicación del IVC a distintos BCR del sudeste bonaerense permitió hacer un primer diagnóstico del estado de conservación de estos ambientes para, en un futuro, implementar medidas de conservación, manejo integral o restauración. Estas medidas podrían ir acompañadas de un programa para dejar de cultivar los BCR, promoviendo iniciativas de reintroducción de plantas nativas y manejando la vegetación espontánea, por ejemplo, a través del control de especies como festuca. Esto es posible en el marco de herramientas como el ordenamiento territorial rural del partido de Balcarce, que cuenta con un programa denominado "Valoración de la naturaleza y los servicios ecosistémicos", que pretende, entre otros objetivos, desarrollar un plan de manejo y restauración de estos elementos del paisaje.

Una futura versión de la encuesta podría contemplar ampliar la aplicación del IVC desarrollado, incluyendo nuevos atributos en el AD e incorporando encuestados de otras áreas geográficas con otras problemáticas ambientales y tipos de ecosistemas. Esto permitiría agregar otros criterios que contribuyen a la funcionalidad ecológica de los BCR, así como sus valores umbrales, que son contexto-dependientes y que pueden variar para diferentes zonas. Por este motivo, varios de los expertos no respondieron a la consulta sobre los valores umbrales y se debería tener en cuenta para mejorar el IVC. Incluso, se podrían incorporar preguntas orientadas a la toma de decisiones y sumar expertos del área política e institucional (e.g., ONGs, el Ministerio de Ambiente, las municipalidades, el Organismo Provincial para el Desarrollo Sostenible de la Provincia de Buenos Aires, etc.). Esto último nos permitiría obtener una opinión experta sobre la factibilidad de aplicar el IVC como una herramienta para el ordenamiento territorial y de las limitaciones que podrían surgir durante el proceso (e.g., presentación de la propuesta a las instituciones, disponibilidad de recursos humanos y económicos, etc.). Finalmente, también se espera ampliar el número de casos de aplicación del IVC en el sudeste bonaerense para tener mayor representatividad de los tipos de BCR existentes en la actualidad.

Agradecimientos. Agradecemos a los 14 profesionales que respondieron la encuesta, y a las Ingenieras Agrónomas Verónica Ispizúa y Eugenia Garavano por su ayuda en la identificación de las especies vegetales. Este trabajo contó con el apoyo financiero del Instituto Nacional de Tecnología Agropecuaria (INTA), Proyectos "Observatorios de Sustentabilidad Rural" PNNAT 1128035 y Evaluación y manejo de la biodiversidad y los servicios ecosistémicos en sistemas productivos" PNNAT 1128053" . 


\section{REFERENCIAS}

Alonso, S. I., and A. Peretti. 2006. Malezas plaga de la agricultura argentina, catálogo de semillas y plántulas. Segunda edición. UNMdP, Buenos Aires, Argentina.

Andrade, A., and R. Vides. 2010. Enfoque Ecosistémico y políticas públicas: aportes para la conservación de la biodiversidad y la adaptación al cambio climático en Latinoamérica. IAI-CIIFEN-MacArthur Foundation, São José dos Campos, Brasil.

Arachevaleta, M., C. W. Bacon, C. S. Hoveland, and D. E. Radcliffe. 1989. Effect of the tall fescue endophyte on plant response to environmental stress. Agronomy Journal 81:83-90.

Barral, M. P., and N. O. Maceira. 2012. Land use planning based on ecosystem service assessment: A case study in the Southeast Pampas of Argentina. Agriculture Ecosystem and Environment 154:34-43.

Belesky, D. P., and J. M. Fedders. 1996. Does endophyte influence regrowth of tall fescue? Annals of Botany 78:499505.

Benton, T. G., J. A. Vickery, and J. D. Wilson. 2003. Farmland biodiversity: is habitat heterogeneity the key? TEE 18: 182-188.

Bilenca, D., M. Codesido, C. G. Fischer, and L. P. Carusi. 2009. Impactos de la actividad agropecuaria sobre la biodiversidad en la ecorregión pampeana. INTA, Buenos Aires, Argentina.

Bilenca, D., C. Codesido, C. Fischer, L. Pérez Carush, E. Zufiaurre, and A. Abba. 2012. Impactos de la transformación agropecuaria sobre la biodiversidad en la provincia de Buenos Aires. Revista del Museo Argentino de Ciencias Naturales 14:189-198.

Bilenca, D., and F. Miñarro. 2004. Identificación de Áreas Valiosas de Pastizal (AVPs) en las Pampas y Campos de Argentina, Uruguay y sur de Brasil. Fundación Vida Silvestre Argentina, Buenos Aires, Argentina.

Burel, F., J. Baudry, A. Butet, P. Clergeau, Y. Delettre, D. Le Coeur, F. Dubs, N. Morvan, G. Paillat, S. Petit, C. Thenail, E. Brunel, and J. Lefeuvre. 1998. Comparative biodiversity along a gradient of agricultural landscapes. Acta Oecologica 19:47-60.

Burkhard, B., F. Kroll, S. Nedkov, and F. Müller. 2012. Mapping supply, demand and budgets of ecosystem services. Ecological Indicators 21:17-29.

Cabrera, A. 1963-70. Flora de la Provincia de Buenos Aires. Colección Científica del INTA, Buenos Aires, Argentina.

Campos, R. E. 2010. Eryngium (Apiaceae) phytotelmata and their macro-invertebrate communities, including a review and bibliography. Hydrobiologia 652:311-328.

Chaneton, E. J., S. B. Perelman, M. Omacini, and R. J. León. 2002. Grazing, environmental heterogeneity, and alien plant invasions in temperate Pampa grasslands. Biological Invasions 4:7-24.

D'Acunto, L., M. Semmartin, and C. M. Ghersa. 2014: Uncropped field margins to mitigate soil carbon losses in agricultural landscapes. Agriculture Ecosystem and Environment 183:60-68.

Delucchi, G. 2006. Las especies vegetales amenazadas de la Provincia de Buenos Aires: Una actualización. APRONA Boletines Científicos 39:19-31.

Duelli, P., and M. K. Obrist. 2003. Biodiversity indicators: the choice of values and measures. Agriculture Ecosystem and Environment 98:87-98.

Elizalde, J. H., and V. H. Lallana. 2000. Revisión sobre aspectos bio-ecológicos de especies del género Eryngium (Apiaceae). Revista de la Facultad de Agronomía UBA 20:269-279.

Forman, R. T., and M. Godron. 1986. Landscape ecology. John Wiley and Sons, NewYork.

Forman, R. T. T., D. Sperling, J. A. Bissonette, A. P. Clevenger, C. D. Cutshall, V. H. Dale, L. Fahrig, R. France, C. R. Goldman, K. Heanue, J. A. Jones, F. J. Swanson, T. Turrentine, and T. C. Winter. 2003. Road Ecology: Science and Solutions. Island Press, Washington, D. C., USA.

Frangi, J. 1975. Sinopsis de las comunidades vegetales y el medio de las sierras de Tandil (Provincia de Buenos Aires). Boletín de la Sociedad Argentina de Botánica 16:29-319.

Gelbard, J., and J. Belnap. 2003. Roads as conduits for exotic plant invasions in a semiarid landscape. Conservation Biology 17:420-432.

Groom, M. J. 2006.Threats to biodiversity. Pp. 63-109 in M. J. Groom, G. K. Meffe and C. R. Carroll (eds.). Principles of conservation biology. Sinauer Associates Inc. Sunderland, Massachusetts, USA.

Herrera, L., and P. Laterra. 2011. Relative influence of disturbance histories and landscape patterns on floristic structure and diversity of fragmented grasslands. Applied Vegetation Science 14:181-188.

Hess, G. R., and R. A. Fischer. 2001. Communicating clearly about conservation corridors. Landscape Urban and Planning 55:195-208.

INTA. 1991. Cartas de suelo de la República Argentina, E 1:50000, INTA.

Isasi-Catalá, E. 2011. Los conceptos de especies indicadoras, paraguas, banderas y claves: su uso y abuso en ecología de la conservación. Interciencia 36:31-38.

Kitching, R. L. 2000. Food webs and container habitats. The Natural History and Ecology of Phytotelmata. Cambridge University Press, Cambridge.

Kuhnert, P. M., T. G. Martin, and S. P. Griffiths. 2010. A guide to eliciting and using expert knowledge in Bayesian ecological models. Ecology Letters 13:900-914.

Landsberg, J., and G. Crowley. 2004. Monitoring rangeland biodiversity: Plants as indicators. Austral Ecology 29: 
59-77.

Laurance, W., and E. Yensen. 1991. Predicting the impacts of edge effects in fragmented habitats. Biological Conservation 55:77-92.

Martin, T. G., M. A. Burgman, F. Fidler, P. M. Kuhnert, S. Low-Choy, M. McBride, and K. Mengersen. 2012. Eliciting expert knowledge in conservation science. Conservation Biology 26:29-38.

Maceira, N., J. Elverdin, C. Álvarez, C. Videla, K. Zelaya, L. Picone, G. Borras, J. Tribo, N. Romera, C. Muñoz, M. Bruno, G. S. Cambareri, E. Álvarez Manzaneda, C. Cufré, M. F. González, P. H. Martínez Melo, L. Herrera, et al. 2011. Proceso participativo para el desarrollo de un Plan de Ordenamiento Territorial Rural del Partido de Balcarce. Memoria Encuentro Nacional de ProFeder: El valor agregado del trabajo conjunto. Coordinador AdrianGargicevich. Ediciones INTA, Argentina.

MAGyP. 2012. Bases para el ordenamiento del territorio rural argentino. URL: ced.agro.uba.ar/ubatic/sites/default/ files/files/libro_ordenamiento_territorial_baja.pdf

Montes, L., S. Alonso, M. Nuciari, A. Clausen, I. Guma, and A. Echarte. 2001. Flora espontánea del sudeste bonaerense: clave ilustrada para la identificación de las principales dicotiledóneas herbáceas por sus caracteres vegetativos. UNMdP, Buenos Aires, Argentina.

Morelli, F., M. Beim, L. Jerzak, D. Jones, and P. Tryjanowski. 2014. Can roads, railways and related structures have positive effects on birds? - A review. Transportation Research Part D 30:21-31.

Mueller-Dombois, D., and H. Ellemberd. 1974. Aims and methods of vegetation ecology. Wiley and Son. New York, USA.

Muller, F. 2005. Indicating ecosystem and landscape organization. Ecological Indicators 5:280-294.

Oesterheld, M. 2008. Impacto de la agricultura sobre los ecosistemas: Fundamentos ecológicos y problemas más relevantes. Ecología Austral 18:337-346.

Paruelo, J. M., J. P. Guerschman, G. Piñeiro, E. G. Jobbágy, S. R. Verón, G. Baldi, and S. Baeza. 2006. Cambios en el uso de la tierra en Argentina y Uruguay: marcos conceptuales para su análisis. Agrociencia 10:47-61.

Poggio, S., E. Chaneton, and M. Ghersa. 2010. Landscape complexity differentially affects alpha, beta, and gamma diversities of plants occurring in fencerows and crop fields. Biological Conservation 143:2477-2486.

Rapoport, E., A. Marzoca, and B. Drausal. 2009. Malezas comestibles del Cono sur. Ediciones INTA, Buenos Aires, Argentina.

Richardson, D. M., P. Pyßek, M. Rejmánek, M. G. Barbour, F. D. Panetta, and C. J. West. 2000. Naturalization and invasion of alien plants: concepts and definitions. Diversity and Distribution 6:93-107.

Roitman, G., and P. Preliasco. 2012. Guía de reconocimiento de herbáceas de la Pampa deprimida. Características para su manejo. Buenas prácticas para una ganadería sustentable de pastizal. Primera edición. Fundación Vida Silvestre Argentina, Aves Argentinas, Buenos Aires, Argentina.

Sabatino, M., N. Maceira, and A. Rovere. 2015. Germinación de Eryngium regnellii: especie clave para la restauración ecológica de las interacciones planta-polinizador en la Pampa Austral (Buenos Aires, Argentina). Revista Internacional de Botánica Experimental Phyton 84:435-443.

Sáez, A., M. Sabatino, and M. Aizen. 2014. La diversidad floral del borde afecta la riqueza y abundancia de visitantes florales nativos en cultivos de girasol. Ecología Austral 24:94-102.

Saunders, D. A., and R. J. Hobbs (eds.). 1991. Nature Conservation 2: The Role of Corridors. Surrey Beatty and Sons, Chipping Norton.

Scheneiter, J. O. 2005. Manejo de Pasturas de Festuca Alta. Documento de resumen. Jornada de campo: Avance en producción y manejo de pasturas. EEA. INTA Pergamino. Buenos Aires, Argentina. URL: www.produccionanimal.com.ar.

Scheneiter, J. O., I. I. Kaufmann, A. R. Ferreyra, and R. T. Llorente. 2015. The herbage productivity of tall fescue in the Pampas region of Argentina is correlated to its ecological niche. Grass and Forage Science 71:403-412.

Sharifi, M. A., L. Boerboom, K. B. Shamsudin, and L. Veeramuthu. 2006. Spatial multiple criteria decision analysis in integrated planning for public transport and land use development study in Klang Valley, Malaysia, ISPRS Technical Commission II Symposium, Vienna. URL: www.isprs.org/proceedings/XXXVI/part2/pdf/sharifi.pdf.

Soriano, A., R. J. León, O. E. Sala, R. S. Lavado, V. A. Deregibus, M. A. Cauéphé, O. A. Scaglia, C. A. Velázquez, and J. H. Lemcoff. 1991. Río de la Plata Grasslands. Pp. 367-407 in R. T. Coupland (ed.). Natural Grasslands. Introduction and Western Hemisphere. Ecosystems of the World, Elsevier, New York.

Sutherland, W. J. 2006. Predicting the ecological consequences of environmental change: a review of the methods. Journal of Applied Ecology 43:599-616.

Teli, S., and P. Kanikar. 2015. A Survey on Decision Tree Based Approaches in Data Mining. IJARCSSE 5:613-617.

Tiemeyer, B., P. Kahle, and B. Lennartz. 2006. Nutrient losses from artificially drained catchments in North-East Germany at different scales. Agricultural Water Management 85:47-57.

Torreta, J. P., and S. L. Poggio. 2013. Species diversity of entomophilous plants and flower-visiting insects is sustained in the field margins of sunflower crops. Journal of Natural History 47:139-165.

van der Ree, R., D. J. Smith, and C. Grilo (eds.). 2015. Handbook of Road Ecology. John Wiley and Sons, Ltd.

Vázquez, P., M. Calandroni, F. Cabria, M. Agnusdei, and M. C. Rojas. 2014. Patrones de índice verde normalizado (IVN) en pastizales de la provincia de Buenos Aires: su asociación con comunidades vegetales y suelos. Ecología Austral 24:276-285.

Vélez-Pareja, I. 2003. Sequential Decisions: Decision Trees (árboles de decisión). URL: ssrn.com/abstract=986975.

Vervoorts, F. 1967. Las comunidades vegetales de la Depresión del Salado. La vegetación de la República Argentina, Serie Fitogeográfica N7 INTA, Buenos Aires, Argentina.

Viglizzo, E. F., F. Lértora, A. J. Pordomingo, J. N. Bernardos, Z. E. Roberto, and H. Del Valle. 2001. Ecological lessons and applications from one century of low external-input farming in the pampas of Argentina. Agriculture, Ecosystems and Environment 83:65-81. 